Featured Article

\title{
How skilled are skilled facilities? Post-discharge complications after colorectal cancer surgery in the U.S.
}

\author{
Mohamed A. Abd El Aziz ${ }^{1}$, Fabian Grass ${ }^{1}$, Kevin T. Behm, Anne-Lise D’Angelo, \\ Kellie L. Mathis, Eric J. Dozois, David W. Larson* \\ Division of Colon and Rectal Surgery, Department of Surgery, Mayo Clinic, Rochester, MN, USA
}

\section{A R T I C L E I N F O}

\section{Article history:}

Received 26 October 2020

Received in revised form

1 December 2020

Accepted 4 December 2020

\section{Keywords:}

Inter-hospital transfer

Discharge destination

Skilled care

Unskilled care

Colorectal surgery

\begin{abstract}
A B S T R A C T
Background: Characteristics and indications for discharging patients to home or a specific facility type have been studied; however, critical evaluation of these facilities through analysis of post-discharge complications and readmission rates is mandatory. The aim of this study was to compare complications occurring after discharge to home, skilled, and unskilled care facilities to identify potential pitfalls. Methods: All adult ( $\geq 18$ years) patients who underwent surgery for colon or rectal cancer from 2012 to 2017 as reported in the American College of Surgeons National Surgical Quality Improvement Program (ACS-NSQIP) database were included. Patients were categorized according to the discharge destination into: home, skilled care (rehabilitation center, separate acute care, skilled facility), and unskilled care (multilevel senior community, facility which is home, unskilled facility). Demographics, surgical risk factors and predischarge complications were compared between the three groups. Primary endpoints were overall, major, surgical, and medical complications occurring post-discharge, within 30 days of surgery. Further assessed were specific complications, readmission, length of stay, and 30-day mortality. Results: A total of 108,617 patients were identified. Of them, 100,478 (92\%) discharged to home, 7313 (7\%) to skilled, and 826 (1\%) to unskilled care. Of patients discharged to skilled care, 1928 (26\%) discharged to rehabilitation centers, 368 (5\%) to separate acute care, and 5017 (69\%) to skilled facilities. Adjusted overall, major, surgical, and medical post-discharge complications were highest among patients discharged to skilled care destinations. Subgroup analysis revealed separate acute care (inter-hospital transfer) to be associated with the highest morbidity. Main reasons for readmission were primarily related to surgical site infection and intestinal obstruction among the three main destinations, whereas readmissions for systemic sepsis and medical complications were more frequent in patients admitted to skilled care.

Conclusion: This study identified higher rates of post-discharge complications associated with skilled care destinations, despite risk adjustment. This over-morbidity is potentially related to prevailing medical complications and inter-hospital transfers. Further studies are needed to better understand those findings and to improve quality of post-acute care and related outcomes.
\end{abstract}

(C) 2020 Published by Elsevier Inc.

\section{Introduction}

Skilled care facilities are licensed healthcare residences providing both short term rehabilitation and long term care for patients with complex medical needs. Post-acute care is important to unburden acute care hospitals given the growing focus on

\footnotetext{
* Corresponding author. Chair of Colon and Rectal Surgery Department of Surgery, Mayo Clinic, 200 first St. Southwest, Rochester, MN, 55905, USA.

E-mail address: Larson.David2@mayo.edu (D.W. Larson).

1 Shared first authorship.
}

financial considerations in a value-based health-care market. ${ }^{1}$ However, recent studies demonstrated conflicting results regarding quality of care and related outcomes of patients admitted to skilled care, especially with regards to the quality-metric of unplanned readmission. ${ }^{2-4}$

Comparison of facilities for quality assessment requires thorough risk stratification considering patient characteristics, surgical management and postoperative recovery including pre-discharge complications. ${ }^{5}$ While several studies aimed to define criteria and indications for discharging patients to home or a specific facility type, critical evaluation of these facilities through analysis of post- 
discharge complications and readmission rates is important to identify potential quality improvement initiatives. ${ }^{6,7}$ National datasets such as the American College of Surgeons National Surgical Quality Improvement Program (ACS-NSQIP) represent a platform for such comparative analysis given the massive data pool and the focus on short-term morbidity through highly standardized outcome assessment and definitions. Furthermore, ACS-NSQIP stratifies facilities further according to level of care in skilled and unskilled facility types.

This six-year longitudinal study compared complications occurring after discharge to home, skilled and unskilled care facilities in order to identify negative trends and potential pitfalls.

\section{Material and methods}

\section{Data source}

The ACS-NSQIP database was queried to include all patients who underwent an elective (defined as patient presented to planned surgery from home) surgery for colon or rectal cancer between 2012 and 2017.

ACS-NSQIP is a national quality improvement program gathering abstract patient information through predesigned data extraction sheets managed by trained data abstractors from all participating institutions. Overall, a random sample of $20 \%$ of the pooled patients from the participating institutions is included in the final Participant User Files (PUF).

After merging the PUF files from 2012 to 2017, patients who underwent elective surgery for colon or rectal cancer were selected using the Current Procedure Terminology (CPT) codes (44140, 44141, 44143, 44144, 44145, 44146, 44147, 44150, 44151, 44155, 44156, 44157, 44158, 44160, 44188, 44204, 44205, 44206, 44207, 44208, 44210, 44211, 44212, 45110, 45111, 45112, 45113, 45114, $45116,45119,45120,45121,45123,45126,45395,45397)$ and the International Classification of Disease (ICD) medical codes (for colon cancer, ICD-9-CM: 153. $\mathrm{x}$ and ICD-10-CM: C18. x; and for rectal cancer (ICD-9-CM: 154. $\mathrm{x}$ and ICD-10-CM: C19, and C20).

\section{Discharge destinations}

According to ACS-NSQIP definitions, discharge destinations were categorized into three main groups: home, skilled care and unskilled care. Patients who discharged against medical advice, discharged to hospice, expired discharge or unknown discharge destination were excluded. Skilled care destinations included the following: separate acute care, skilled facility, and rehabilitation center. Unskilled care destinations were subcategorized as follows: multilevel senior community, facility which is home, unskilled facility.

\section{Covariates and outcomes}

Baseline characteristics and perioperative information were compared between the three main groups. Primary endpoints were overall, surgical or medical complications occurring within 30 days of the index operation. Secondary endpoints were specific complications according to standardized ACS NSQIP definitions including surgical site infection (SSI), wound disruption, need for blood transfusion, systemic sepsis, respiratory complications (pneumonia, unplanned intubation, and/or mechanical ventilation $\geq 48 \mathrm{~h}$ ), renal complications (acute renal failure and/or progressive renal insufficiency), urinary tract infection (UTI), vascular thromboembolism (VTE, including pulmonary embolism (PE) and/or deep venous thrombosis (DVT)), and major adverse cardiovascular events (MACE, including stroke, myocardial infarction, and/or cardiac arrest requiring cardiopulmonary resuscitation). Further assessed were unplanned reoperation, readmission and mortality within 30 days of surgery.

Specific reasons for unplanned readmission from home or skilled/unskilled care destinations were categorized, summarized and compared.

All outcomes were assessed in-hospital before discharge (predischarge complications, until 1st discharge after index surgery) and after discharge (post-discharge complications, until postoperative day (POD) 30).

Subgroup analysis of patients discharged to skilled care destinations was performed to further detail post-discharge morbidity associated with separate acute care, skilled facilities, and rehabilitation centers.

\section{Statistical analysis}

The descriptive data were summarized using frequencies and percentages for categorical data and median (interquartile range) for continuous data. Chi-Square test was used to assess the differences between the three groups for categorical data and the Independent Samples Kruskal Wallis test was used for continuous data. Outcomes that had a p-value $<0.1$ after univariate analysis were further assessed through multivariable analysis to adjust for the baseline confounders. A p-value $<0.05$ was considered statistically significant and all tests were two-sided.

The analysis was performed using the Statistical Package for Social Sciences (SPSS, Version 25; SPSS, Inc., Armonk, NY, USA).

a) Overall

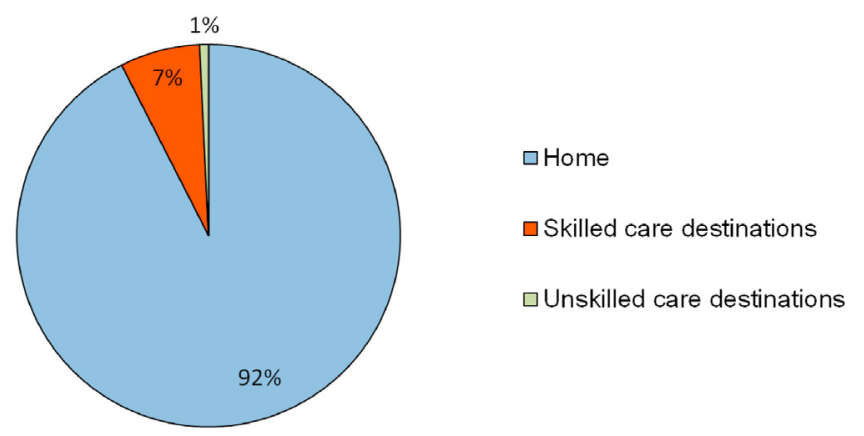

b) Skilled care destinations

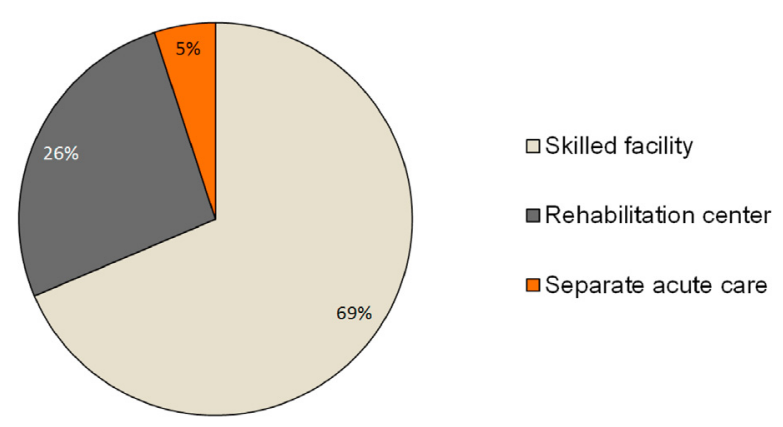

Fig. 1. Discharge destination.

Percentages of different discharge destinations a) overall and b) among skilled care destinations. 
Table 1

Baseline demographics and surgical specifics.

\begin{tabular}{|c|c|c|c|c|c|}
\hline & Home $\mathrm{N}=100478$ & Skilled N = 7313 & Unskilled $\mathrm{N}=826$ & Total $\mathrm{N}=108617$ & P value ${ }^{a}$ \\
\hline Age, Median (IQR) & $65(55-74)$ & $79(70-85)$ & $72(60-83)$ & $66(56-75)$ & $<0.0001$ \\
\hline Age $>80$ years & $10760(10.7 \%)$ & $3114(42.6 \%)$ & $264(32.0 \%)$ & $14138(13.0 \%)$ & $<0.0001$ \\
\hline Male & $54154(53.9 \%)$ & 3395 (46.4\%) & 446 (54.0\%) & $57995(53.4 \%)$ & $<0.0001$ \\
\hline ASA class & & & & & $<0.0001$ \\
\hline $1-2$ & 42477 (42.3\%) & $1090(14.9 \%)$ & $165(20.0 \%)$ & $43732(40.3 \%)$ & \\
\hline$\geq 3$ & $57871(57.6 \%)$ & $6219(85.0 \%)$ & $659(79.8 \%)$ & 64749 (59.6\%) & \\
\hline Not assigned & $130(0.1 \%)$ & $4(0.1 \%)$ & $2(0.2 \%)$ & $136(0.1 \%)$ & \\
\hline \multicolumn{6}{|l|}{ BMI, $\mathrm{kg} / \mathrm{m}^{2}$} \\
\hline Median (IQR) & $27.7(24.2-32)$ & $27(23-32)$ & $27(24-31)$ & $28(24-32)$ & $<0.0001$ \\
\hline $\mathrm{DM}$ & $18388(18.3 \%)$ & $1976(27.0 \%)$ & $176(21.3 \%)$ & $20540(18.9 \%)$ & $<0.0001$ \\
\hline Smoker & $14339(14.3 \%)$ & $805(11.0 \%)$ & $119(14.4 \%)$ & $15263(14.1 \%)$ & $<0.0001$ \\
\hline Dyspnea & $6944(6.9 \%)$ & $1154(15.8 \%)$ & $93(11.3 \%)$ & $8191(7.5 \%)$ & $<0.0001$ \\
\hline Functional health status: & & & & & $<0.0001$ \\
\hline Independent & 99169 (98.7\%) & $6538(89.4 \%)$ & $614(74.3 \%)$ & $106321(97.9 \%)$ & \\
\hline Dependent & $975(1.0 \%)$ & $730(10.0 \%)$ & $203(24.6 \%)$ & $1908(1.8 \%)$ & \\
\hline Unknown & $334(0.3 \%)$ & $45(0.6 \%)$ & $9(1.1 \%)$ & $388(0.4 \%)$ & \\
\hline COPD & $4314(4.3 \%)$ & $838(11.5 \%)$ & $69(8.4 \%)$ & $5221(4.8 \%)$ & $<0.0001$ \\
\hline Ascites & $202(0.2 \%)$ & $39(0.5 \%)$ & $2(0.2 \%)$ & $243(0.2 \%)$ & $<0.0001$ \\
\hline $\mathrm{CHF}$ & $607(0.6 \%)$ & $173(2.4 \%)$ & $15(1.8 \%)$ & $795(0.7 \%)$ & $<0.0001$ \\
\hline HTN & $50728(50.5 \%)$ & $5147(70.4 \%)$ & $501(60.7 \%)$ & $56376(51.9 \%)$ & $<0.0001$ \\
\hline Currently on dialysis & $350(0.3 \%)$ & $78(1.1 \%)$ & $9(1.1 \%)$ & $437(0.4 \%)$ & $<0.0001$ \\
\hline Disseminated cancer & $9309(9.3 \%)$ & $723(9.9 \%)$ & $69(8.4 \%)$ & $10101(9.3 \%)$ & 0.135 \\
\hline Open wound/wound infection & $483(0.5 \%)$ & $142(1.9 \%)$ & $15(1.8 \%)$ & $640(0.6 \%)$ & $<0.0001$ \\
\hline Chronic steroid use & $2814(2.8 \%)$ & $315(4.3 \%)$ & $24(2.9 \%)$ & $3153(2.9 \%)$ & $<0.0001$ \\
\hline Loss of body weight ( $>10 \%$ within last 6 months) & $4065(4.0 \%)$ & $485(6.6 \%)$ & $46(5.6 \%)$ & $4596(4.2 \%)$ & $<0.0001$ \\
\hline Bleeding disorder & $2369(2.4 \%)$ & $411(5.6 \%)$ & $36(4.4 \%)$ & $2816(2.6 \%)$ & $<0.0001$ \\
\hline Transfusion of $\geq 1 \mathrm{pRBC} / 72 \mathrm{~h}$ before the surgery & $753(0.7 \%)$ & $182(2.5 \%)$ & $28(3.4 \%)$ & $963(0.9 \%)$ & $<0.0001$ \\
\hline Underlying disease & & & & & $<0.0001$ \\
\hline Colon cancer & $66829(66.5 \%)$ & $4989(68.2 \%)$ & $616(74.6 \%)$ & $72434(66.7 \%)$ & \\
\hline Rectal cancer & $33649(33.5 \%)$ & $2324(31.8 \%)$ & $210(25.4 \%)$ & $36183(33.3 \%)$ & \\
\hline \multicolumn{6}{|l|}{ Preoperative laboratories, Median (IQR) } \\
\hline Albumin, g/dl, & $4(3.6-4.3)$ & $3.6(3.2-4)$ & $3.6(3.2-4)$ & $3.9(3.6-4.2)$ & $<0.0001$ \\
\hline Hematocrite, \% & $38.2(34.2-41.7)$ & $34.8(31-41.9)$ & $35.4(30.6-39.4)$ & $38.2(34-41)$ & $<0.0001$ \\
\hline Platelets, k/mL & $250(203-309)$ & $248(196-319)$ & $256(195-330)$ & $249(202-308)$ & 0.172 \\
\hline Operative approach & & & & & $<0.0001$ \\
\hline Minimally invasive & $65038(64.7 \%)$ & 3497 (47.8\%) & $443(53.6 \%)$ & $68978(63.5 \%)$ & \\
\hline Open & $35440(35.3 \%)$ & $3816(52.2 \%)$ & $383(46.4 \%)$ & $39639(36.5 \%)$ & \\
\hline Extent of resection & & & & & $<0.0001$ \\
\hline Segmental colectomy & $65027(64.7 \%)$ & $5170(70.7 \%)$ & $626(75.8 \%)$ & $70823(65.2 \%)$ & \\
\hline Total colectomy & $1775(1.8 \%)$ & $175(2.4 \%)$ & $8(1.0 \%)$ & $1958(1.8 \%)$ & \\
\hline proctectomy & $33676(33.5 \%)$ & $1968(26.9 \%)$ & $192(23.2 \%)$ & $35836(33.0 \%)$ & \\
\hline Diversion & & & & & $<0.0001$ \\
\hline \multicolumn{6}{|l|}{ Yes } \\
\hline Ileostomy & $4349(4.3 \%)$ & $322(4.4 \%)$ & $18(2.2 \%)$ & $4689(4.3 \%)$ & \\
\hline Colostomy & $16549(16.5 \%)$ & $1793(24.5 \%)$ & $166(20.1 \%)$ & $18508(17.0 \%)$ & \\
\hline No & 77279 (76.9\%) & $5064(69.2 \%)$ & $626(75.8 \%)$ & $82969(76.4 \%)$ & \\
\hline Unknown & $2301(2.3 \%)$ & $134(1.8 \%)$ & $16(1.9 \%)$ & $2451(2.3 \%)$ & \\
\hline Operation time (minutes), Median (IQR) & $178(124-253)$ & $179(119-265)$ & $158(111-233)$ & $178(124-253)$ & $<0.0001$ \\
\hline
\end{tabular}

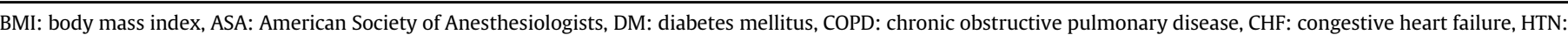
hypertension requiring medication, pRBC: packed red blood cells.

a Derived from Chi-square for categorical variables and Independent Samples Kruskal Wallis test for continuous variables.

\section{Results}

A total of 108,617 patients were included and discharged to home, skilled care and unskilled care, as detailed in Fig. 1. Skilled care destinations ( $\mathrm{n}=7313$ ) included skilled facilities $(69 \%)$, rehabilitation centers (26\%), and separate acute care (5\%). Unskilled care destinations $(\mathrm{n}=826)$ included facilities which were home (83\%), unskilled facilities which were not home (16\%), and multilevel senior communities (1\%).

Demographics, comorbidities, and surgical specifics including approach, extent of resection, ostomy creation, and duration of surgery are detailed in Table 1 , revealing important differences regarding age, dependence status, co-morbidities, and surgeryrelated items between the main three groups (home vs. skilled care vs. unskilled care). In-hospital complications occurring before 1st discharge after the primary surgery and index length of stay are specified in Table 2. In general, rates of overall, major, surgical, medical, and specific complications were highest in patients discharged to skilled care and lowest in patients discharged to home.

\section{Post-discharge complications}

Rates of post-discharge complications occurring in different post-acute care settings are illustrated in Fig. 2. Significant differences between the three main groups were observed for overall, major, surgical and medical complications, which were highest in skilled care facilities and lowest in patients discharged to home. Thirty-day mortality was $0.3 \%$ (374/108,607 patients) overall and $0.2 \%$ in patients discharged to home, $1.8 \%$ in patients discharged to skilled care and $1.1 \%$ in patients discharged to unskilled care (Fig. 2a). Odds to complications after adjustment for the baseline confounders are displayed in Fig. 2b. No significant differences were observed between home vs. unskilled care destinations, whereas all differences were significant comparing home vs. skilled 
Table 2

Pre-discharge postoperative complications.

\begin{tabular}{|c|c|c|c|c|c|}
\hline & $\begin{array}{l}\text { Home } \\
\mathrm{N}=100478\end{array}$ & $\begin{array}{l}\text { Skilled } \\
N=7313\end{array}$ & $\begin{array}{l}\text { Unskilled } \\
\mathrm{N}=826\end{array}$ & $\begin{array}{l}\text { Total } \\
\mathrm{N}=108617\end{array}$ & P value $^{\mathrm{a}}$ \\
\hline Overall morbidity $^{\mathrm{b}}$ & $22792(22.7 \%)$ & $4501(61.5 \%)$ & $358(43.3 \%)$ & $27651(25.5 \%)$ & $<0.0001$ \\
\hline Major complication ${ }^{c}$ & $11415(11.4 \%)$ & $2776(38.0 \%)$ & $205(24.8 \%)$ & $14396(13.3 \%)$ & $<0.0001$ \\
\hline Any Surgical complication & $10912(10.9 \%)$ & $2471(33.8 \%)$ & $184(22.3 \%)$ & $13567(12.5 \%)$ & $<0.0001$ \\
\hline Any SSI & $2650(2.6 \%)$ & $784(10.7 \%)$ & $42(5.1 \%)$ & $3476(3.2 \%)$ & $<0.0001$ \\
\hline Superficial SSI & $1144(1.1 \%)$ & $236(3.2 \%)$ & $16(1.9 \%)$ & $1396(1.3 \%)$ & $<0.0001$ \\
\hline Deep SSI & $236(0.2 \%)$ & $105(1.4 \%)$ & $8(1.0 \%)$ & $349(0.3 \%)$ & $<0.0001$ \\
\hline Organ/Space infection & $1396(1.4 \%)$ & $498(6.8 \%)$ & $21(2.5 \%)$ & $1915(1.8 \%)$ & $<0.0001$ \\
\hline Wound disruption & $235(0.2 \%)$ & $26(1.7 \%)$ & $8(1.0 \%)$ & $369(0.3 \%)$ & $<0.0001$ \\
\hline Need for blood transfusion & $7613(7.6 \%)$ & $1610(22.0 \%)$ & $133(16.1 \%)$ & $9356(8.6 \%)$ & $<0.0001$ \\
\hline Systemic Sepsis & $1250(1.2 \%)$ & $626(8.6 \%)$ & $28(3.4 \%)$ & $1904(1.8 \%)$ & $<0.0001$ \\
\hline Sepsis & $1032(1.0 \%)$ & $331(4.5 \%)$ & $22(2.7 \%)$ & $1385(1.3 \%)$ & $<0.0001$ \\
\hline Septic shock & $221(0.2 \%)$ & $305(4.2 \%)$ & $6(0.7 \%)$ & $532(0.5 \%)$ & $<0.0001$ \\
\hline Unplanned reoperation & $1914(1.9 \%)$ & $695(9.5 \%)$ & $24(2.9 \%)$ & $2633(2.4 \%)$ & $<0.0001$ \\
\hline Any medical complication & $2881(2.9 \%)$ & $1293(17.7 \%)$ & 77 (9.3\%) & $4251(3.9 \%)$ & $<0.0001$ \\
\hline Respiratory complications & $1089(1.1 \%)$ & $785(10.7 \%)$ & $38(4.6 \%)$ & $1912(1.8 \%)$ & $<0.0001$ \\
\hline Pneumonia & $728(0.7 \%)$ & $489(6.7 \%)$ & $29(3.5 \%)$ & $1246(1.1 \%)$ & $<0.0001$ \\
\hline Unplanned intubation & $399(0.4 \%)$ & $366(5.0 \%)$ & $13(1.6 \%)$ & $778(0.7 \%)$ & $<0.0001$ \\
\hline On ventilator for $>48 \mathrm{~h}$ & $199(0.2 \%)$ & $361(4.9 \%)$ & $7(0.8 \%)$ & $567(0.5 \%)$ & $<0.0001$ \\
\hline Renal complications & $350(0.3 \%)$ & $174(2.4 \%)$ & $5(0.6 \%)$ & $529(0.5 \%)$ & $<0.0001$ \\
\hline Progressive renal insufficiency & $271(0.3 \%)$ & $99(1.4 \%)$ & $4(0.5 \%)$ & $374(0.3 \%)$ & $<0.0001$ \\
\hline AKI & $82(0.1 \%)$ & $77(1.1 \%)$ & $1(0.1 \%)$ & $160(0.1 \%)$ & $<0.0001$ \\
\hline UTI & $805(0.8 \%)$ & $259(3.5 \%)$ & $28(3.4 \%)$ & $1092(1.0 \%)$ & $<0.0001$ \\
\hline MACE & 449 (0.4\%) & $239(3.3 \%)$ & $13(1.6 \%)$ & $701(0.6 \%)$ & $<0.0001$ \\
\hline Stroke & $42(0.0 \%)$ & $62(0.8 \%)$ & $6(0.7 \%)$ & $110(0.1 \%)$ & $<0.0001$ \\
\hline Cardiac arrest requiring CPR & $58(0.1 \%)$ & $55(0.8 \%)$ & $2(0.2 \%)$ & $115(0.1 \%)$ & $<0.0001$ \\
\hline MI & $359(0.4 \%)$ & $136(1.9 \%)$ & $5(0.6 \%)$ & $500(0.5 \%)$ & $<0.0001$ \\
\hline VTE & $477(0.5 \%)$ & $207(2.8 \%)$ & $4(0.5 \%)$ & $688(0.6 \%)$ & $<0.0001$ \\
\hline DVT & $286(0.3 \%)$ & $149(2.0 \%)$ & $3(0.4 \%)$ & $438(0.4 \%)$ & $<0.0001$ \\
\hline $\mathrm{PE}$ & $229(0.2 \%)$ & $84(1.1 \%)$ & $3(0.4 \%)$ & $316(0.3 \%)$ & $<0.0001$ \\
\hline LOS, days, Median (IQR) & $4(3-6)$ & $8(5-13)$ & $6(4-9)$ & $4(3-7)$ & $<0.0001$ \\
\hline LOS $>7$ days $(>3$ rd $Q)$ & $16664(16.6 \%)$ & $3822(52.3 \%)$ & $279(33.8 \%)$ & $20765(19.1 \%)$ & $<0.0001$ \\
\hline
\end{tabular}

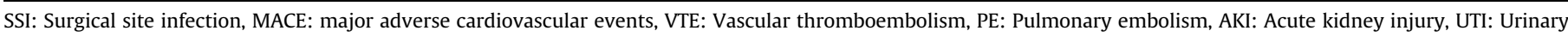
tract infection, CPR: Cardiopulmonary resuscitation, MI: Myocardial infarction, DVT: Deep venous thrombosis, LOS: Length of stay.

a Derived from Chi-square for categorical variables and Independent Samples Kruskal Wallis test for continuous variables.

b Overall morbidity includes any surgical, any medical complications, and/or LOS $>7$ days.

c Major complications include VTE, MACE, renal, respiratory, unplanned reoperation, systemic sepsis, need for blood transfusion, wound disruption, organ space infection and/or deep SSI.

care destinations (all $\mathrm{p}<0.05$ ).

Sub-group analysis of skilled care destinations

Demographic and surgical/postoperative details of the patient population discharged to skilled care are provided in the online appendix. Post-discharge complications (unadjusted and adjusted) related to the three different subgroups of skilled care destinations are detailed in Fig. 3a and b, revealing highest overall, major, surgical, and medical complications associated with separate acute care. After adjustment for the baseline confounders and taking home as a reference, the adjusted OR for 30-day mortality was 2.0; $95 \% \mathrm{CI} 1.2-3.5$ for rehabilitation centers vs. OR $11.7 ; 95 \%$ CI $6.3-21.8$ for separate acute care vs. OR $3.1 ; 95 \%$ CI $2.2-4.3$ for skilled facilities.

Specific surgical and medical complications between the three subgroups of skilled care are presented in Table 3.

\section{Unplanned readmission}

The overall unplanned readmission rate was $8.6 \%$ (8.4\% (home) vs. $11.7 \%$ (skilled care) vs. $10.4 \%$ (unskilled care), $\mathrm{p}<0.0001$ ). Main reasons for readmission are illustrated in Fig. 4. Across all discharge settings, SSI and intestinal obstruction were prevailing reasons. For skilled care destination types, readmission rates were $11.5 \%$ (adjusted OR compared to home 1.1; 95\% CI 0.9-1.4) for rehabilitation centers vs. 9.5\% (adjusted OR 0.9; 95\% CI 0.6-1.4) for separate acute care vs. $11.9 \%$ (adjusted OR 1.3; 95\% CI 1.1-1.4) for skilled facilities Fig. 3.

\section{Discussion}

The present large scale study assessing 30-day morbidity within different post-discharge settings revealed higher post-discharge complication-, readmission- and mortality rates associated with skilled care when compared to unskilled care and home destinations. The main determinants for readmission were primarily related to SSI and intestinal obstruction across all three discharge destinations, whereas readmissions for systemic sepsis and medical complications were more frequent in patients admitted to skilled care. Subgroup analysis of skilled care facility types revealed that (inter-hospital) transfer to separate acute care was associated with the highest morbidity.

This nationwide analysis revealed that $8 \%$ of patients undergoing colorectal surgery are discharged to post-acute care destinations. These patients were older, predominantly functionally dependent, and had more existing comorbidities and higher rates of postoperative complications prior to discharge. Several former studies used large data pools such as ACS-NSQIP to derive prediction models in order to tailor discharge destinations to the individual patient. Mohanty et al. created a surgical risk calculator to accurately predict the risk of discharge to post-acute care settings. ${ }^{8}$ Similarly, Hyder et al. derived and validated a simple patientcentered calculator to predict home discharge after surgery. ${ }^{9}$ More recently, Al-Mazrou et al. stressed the importance of patient-driven factors leading to extended care after colectomy. ${ }^{7}$ 
a) Unadjusted

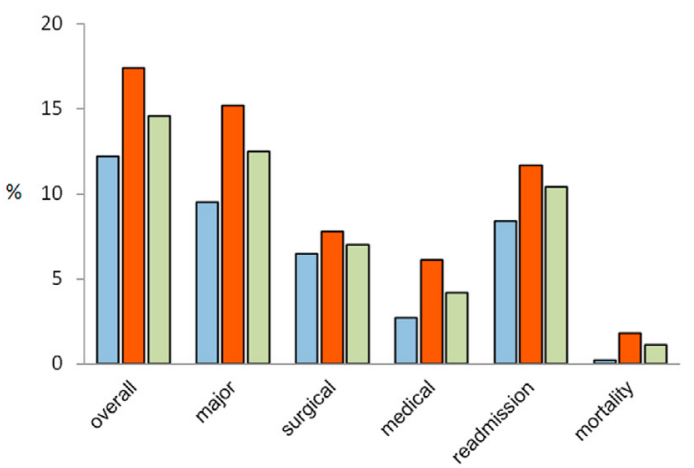

b) Adjusted

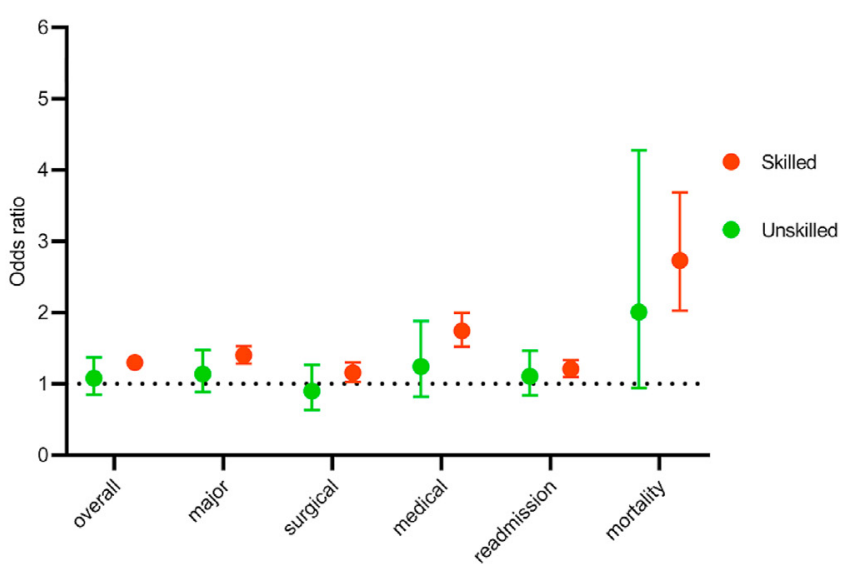

Fig. 2. Post-discharge complications.

Comparison of post-discharge complications of patients discharged to home $(\mathrm{n}=100,478)$, skilled discharge destinations $(\mathrm{n}=7313)$ and unskilled discharge destinations $(\mathrm{n}=826)$ a) unadjusted and $\mathrm{b}$ ) adjusted for: age, gender, race, BMI, diabetes mellitus, current smoker within one year, dyspnea, functional health status prior to surgery, history of severe COPD, ascites, congestive heart failure (CHF) within 30 days before surgery, hypertension requiring medication, hemodialysis, disseminated cancer, open wound/wound infection, steroid use for chronic condition, $>10 \%$ loss body weight in last 6 months, bleeding disorders, transfusion $\geq 1$ unit within $72 \mathrm{~h}$ before surgery, ASA class, underlying disease, pre-operative serum albumin, pre-operative hematocrit, operative approach, diversion, total operation time, days from operation to discharge, any surgical complication before discharge, any medical complication before discharge. Displayed are Odds ratios (dots) with $95 \%$ confidence interval.

Patients discharged to home are used as reference.

While these tools are useful to help with discharge planning, they are unable to assess the outcomes of different post-acute care settings. ${ }^{10}$ This present study used sensitive ACS-NSQIP data stratifying skilled and unskilled facilities in order to focus on postdischarge complications and hospital readmissions. This series attempts to better understand at a macro level the previously described over-morbidity associated with skilled care facilities. $^{1,5,11,12}$

In line with previous investigations, the present study demonstrated higher rates of overall and specific surgical and medical complications associated with post-acute skilled care after riskadjustment for demographic, peri- and postoperative risk profiles. Subgroup analysis of three different skilled care settings revealed a particularly higher rate associated with the small group of patients transferred to separate acute care (Fig. 3b). Inter-hospital transfer has been previously linked to increased morbidity and mortality and may actually penalize hospitals that frequently accept transfers. $^{13-15}$ These findings are likely explained by patients a) Unadjusted

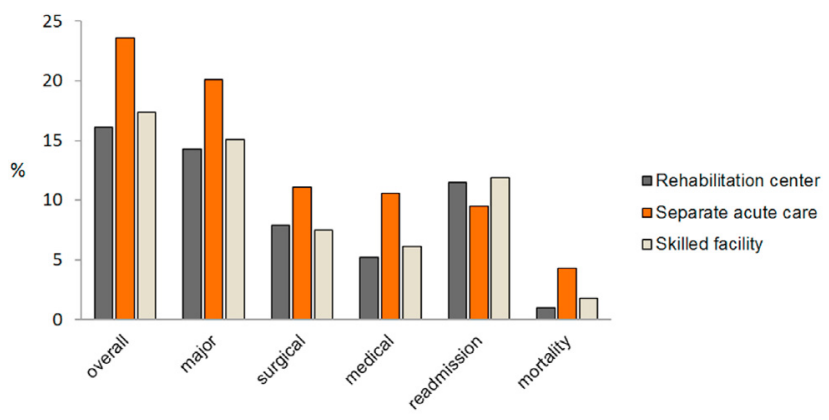

b) Adjusted

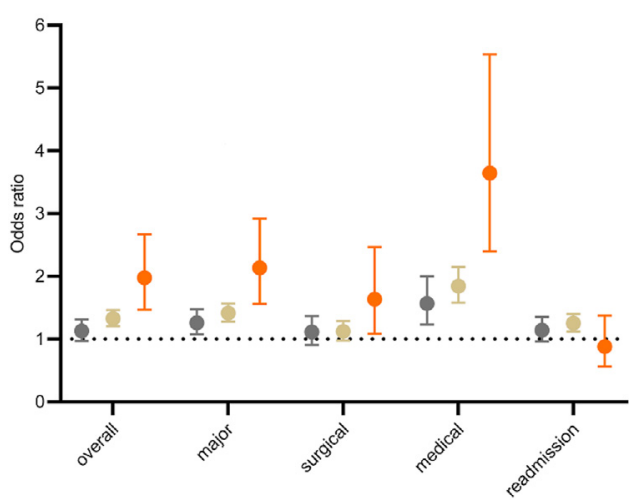

- Rehabiliation center

Skilled facility

Separate acute care

Fig. 3. Post-discharge complications skilled care destinations.

Comparison of post-discharge complications of patients discharged to skilled discharge destinations: rehabilitation center $(n=1928)$, skilled facility $(n=5017)$ and separate acute care $(\mathrm{n}=368)$ a) unadjusted and $\mathrm{b})$ adjusted for: age, gender, race, BMI, diabetes mellitus, current smoker within one year, dyspnea, functional health status prior to surgery, history of severe COPD, ascites, congestive heart failure (CHF) within 30 days before surgery, hypertension requiring medication, hemodialysis, disseminated cancer, open wound/wound infection, steroid use for chronic condition, $>10 \%$ loss body weight in last 6 months, bleeding disorders, transfusion $\geq 1$ unit within $72 \mathrm{~h}$ before surgery, ASA class, underlying disease, pre-operative serum albumin, pre-operative hematocrit, operative approach, diversion, total operation time, days from operation to discharge, any surgical complication before discharge, any medical complication before discharge. Displayed are Odds ratios (dots) with 95\% confidence interval.

Patients discharged to home are used as reference. Mortality not plotted given wide range of $95 \% \mathrm{CI}$.

transferring to larger regional medical centers for higher levels of care after experiencing complications following their index operation. This has to be considered when interpreting morbidity associated with skilled care destinations since inter-hospital transfers are frequently included in this group. ${ }^{6}$

While post-discharge morbidity associated with rehabilitation centers was close or similar to the home population, the large group of patients admitted to dedicated skilled facilities (69\% of skilled facility destinations) exhibited worse outcomes. Specific assessment of post-discharge complications revealed that this increased morbidity was mainly due to medical (adjusted OR $1.8, \mathrm{p}<0.0001$ ) rather than surgical complications (adjusted OR 1.1, n. s.) with one notable exception of septic complications (adjusted OR 1.8, $\mathrm{p}<0.0001$, Table 3 ). These findings suggest that common surgical complications such as SSI and postoperative ileus were safely managed, whereas life-threatening septic complications requiring more invasive treatment represented a challenge. The potential of these complications to overburden the management capacities of skilled facilities represents a serious consideration in our search for value. ${ }^{16,17}$ Among medical complications, respiratory (adjusted OR $2.9, \mathrm{p}<0.0001$ ) issues were the most critical and emphasize the 
Table 3

Multivariable binary logistic regression analysis for specific post-discharge surgical and medical complications of patients admitted to skilled care.

\begin{tabular}{|c|c|c|c|c|c|c|}
\hline & \multicolumn{2}{|l|}{ Rehabilitation center } & \multicolumn{2}{|l|}{ Separate acute care } & \multicolumn{2}{|l|}{ Skilled facility } \\
\hline & Adjusted $\mathrm{OR}^{\mathrm{a}}$ & P value & Adjusted $\mathrm{OR}^{\mathrm{a}}$ & P value & Adjusted $\mathrm{OR}^{\mathrm{a}}$ & $P$ value \\
\hline Any Surgical complication & $1.113(0.906-1.369)$ & 0.308 & $1.634(1.084-2.463)$ & 0.019 & $1.122(0.979-1.286)$ & 0.098 \\
\hline Any SSI & $1.031(0.810-1.312)$ & 0.802 & $1.245(0.742-2.087)$ & 0.407 & $1.020(0.868-1.197)$ & 0.813 \\
\hline Wound disruption & $1.384(0.734-2.611)$ & 0.315 & NA & & $1.103(0.685-1.779)$ & 0.686 \\
\hline Systemic Sepsis & $1.470(0.996-2.171)$ & 0.053 & $2.959(1.545-5.669)$ & 0.001 & $1.815(1.427-2.309)$ & $<0.0001$ \\
\hline Unplanned reoperation & $1.276(0.878-1.855)$ & 0.201 & $2.717(1.502-4.915)$ & 0.001 & $1.028(0.786-1.344)$ & 0.841 \\
\hline Any medical complication & $1.567(1.230-1.997)$ & $<0.0001$ & $3.643(2.398-5.536)$ & $<0.0001$ & $1.843(1.580-2.149)$ & $<0.0001$ \\
\hline Respiratory complications & $2.902(1.852-4.548)$ & $<0.0001$ & $13.5(7.8-23.5)$ & $<0.0001$ & $2.617(1.908-3.589)$ & $<0.0001$ \\
\hline Renal complications & $1.734(1.063-2.829)$ & 0.027 & $3.684(1.587-8.547)$ & 0.002 & $1.692(1.202-2.381)$ & 0.003 \\
\hline UTI & $1.324(0.885-1.980)$ & 0.171 & $1.369(0.504-3.716)$ & 0.538 & $1.738(1.363-2.217)$ & $<0.0001$ \\
\hline MACE & $0.681(0.247-1.880)$ & 0.459 & $5.202(1.863-14.527)$ & 0.002 & $1.944(1.265-2.988)$ & 0.002 \\
\hline VTE & $1.908(1.187-3.067)$ & 0.008 & $3.553(1.550-8.141)$ & 0.003 & $1.626(1.158-2.283)$ & 0.005 \\
\hline
\end{tabular}

OR: Odds Ratio, SSI: Surgical Site Infection, UTI: Urinary Tract Infection, MACE: Major Adverse Cardiovascular Events, VTE: Vascular Thromboembolism.

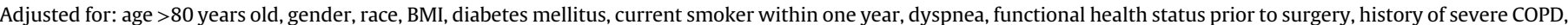

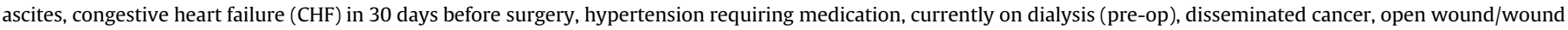

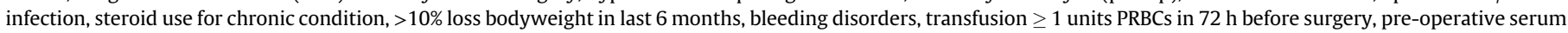

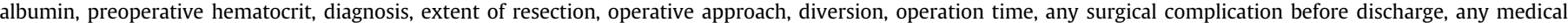
complication before discharge, days from operation to discharge.

a Patients discharged to home were used as a reference.

importance of preventive measures beyond hospital discharge. ${ }^{18}$ Taken together, these results including the associated ovemortality (adjusted OR 3.1, p $<0.0001$ ) suggest that serious complications can occur and if they do require immediate and facilitated transfer to index facilities. ${ }^{4}$

These previous observations were reflected by the detailed analysis of reasons for readmission (Fig. 4), revealing a comparatively higher proportion of patients with septic complications readmitted from skilled care destinations (Fig. 4). On the other hand, readmissions were comparatively lower for patients with intestinal obstruction (postoperative ileus), which may point towards the successful management of these patients in post-acute care settings. The study revealed further dehydration as a prevailing reason for readmission across all discharge destinations in a cohort yielding an ileostomy rate of $4.3 \%$. Surveilling and maintaining hydration status appears to be of critical importance to prevent costly hospital readmissions. ${ }^{19,20}$

The present study also evaluated outcomes of those patients (around 1\% of the total cohort) admitted to unskilled care destinations. At first glance, outcomes appear encouraging and close to the patient population discharged to home. ${ }^{21}$ However, "unskilled care" represents a vague definition, and underreporting of sensitive

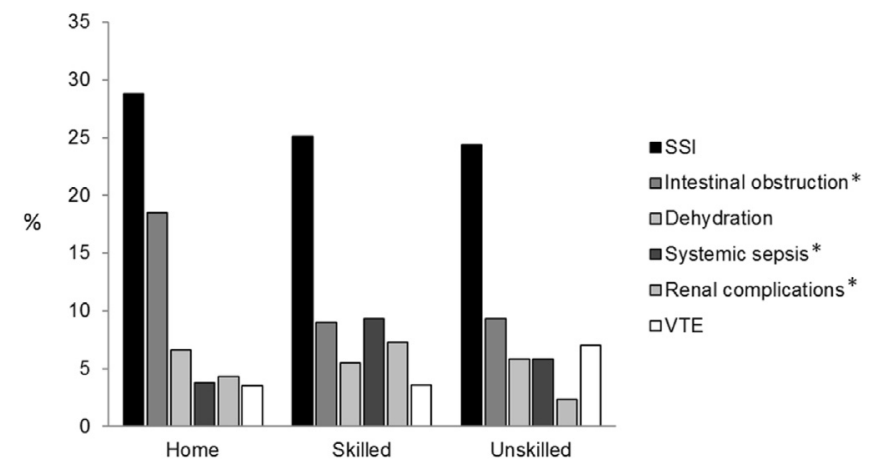

Fig. 4. Reasons for readmission

Most frequent reasons for hospital readmission to index facility from home ( $n=8396$ readmitted patients), skilled care destinations ( $n=852$ readmitted patients) and unskilled care destinations ( $\mathrm{n}=86$ patients).

*represent reasons with statistically significant differences (multigroup comparison; all $\mathrm{p}<0.001$ ).

SSI - any surgical site infection, VTE - venous thromboembolism. outcomes cannot be excluded. ${ }^{22}$ Nevertheless, VTE appears to be higher in unskilled care facilities and was overrepresented among patients readmitted from unskilled care compared to the other settings. This emphasizes the importance of pre-discharge education regarding DVT prophylaxis of this fragile, dependent subset of patients. $^{23}$

Taken together, the present study identified several potential pitfalls associated with skilled and unskilled care settings. These need to be interpreted within the limitations of this study. First, despite risk stratification of outcomes, residual confounding cannot be excluded. There may notably be an important impact of psychosocial components on the choice of discharge destination not accounted for in this analysis. Second, ACS-NSQIP does not provide data on setting and case volume of contributing centers, which may be important since academic centers tend to discharge fewer patients to post-acute care facilities. ${ }^{24}$ Third, categorization of discharge destinations is broad, and further specifics on size, experience, and staffing as quality metrics were not available. Moreover, transfer to a higher level of care may impact the results and thus partly explain the higher complication rates in the skilled group. It is unclear whether the transfer was to a long-term care facility or a higher level of care due to complications. Lastly, details on functional recovery (mobilization, oral intake, fluid balance), arguably impacting post-discharge planning, were not available.

In conclusion, this study identified higher rates of postdischarge complications associated with skilled care destinations, despite risk adjustment. This over-morbidity is potentially related to prevailing medical complications and inter-hospital transfers. Further studies are needed to better understand those findings and to improve quality of post-acute care and related outcomes.

\section{Declaration of competing interest}

None.

\section{Appendix A. Supplementary data}

Supplementary data to this article can be found online at https://doi.org/10.1016/j.amjsurg.2020.12.012.

\section{References}

1. Chatterjee P, Qi M, Werner R. Relative contributions of hospital versus skilled 
nursing facility quality on patient outcomes. BMJ Qual Saf. 2020 [Online ahead of print].

2. Krumholz HM, Wang K, Lin Z, et al. Hospital-readmission risk - isolating hospital effects from patient effects. N Engl J Med. 2017;377(11):1055-1064.

3. Mileski M, Topinka JB, Lee K, Brooks M, McNeil C, Jackson J. An investigation of quality improvement initiatives in decreasing the rate of avoidable 30-day, skilled nursing facility-to-hospital readmissions: a systematic review. Clin Interv Aging. 2017;12:213-222.

4. Paredes AZ, Malik AT, Cluse M, et al. Discharge disposition to skilled nursing facility after emergent general surgery predicts a poor prognosis. Surgery. 2019;166(4):489-495.

5. Neuman MD, Wirtalla C, Werner RM. Association between skilled nursing facility quality indicators and hospital readmissions. J Am Med Assoc. 2014;312(15):1542-1551.

6. Saadat LV, Mahvi DA, Jolissaint JS, Urman RD, Gold JS, Whang EE. Discharge destination following rectal cancer resection: an analysis of preoperative and intraoperative factors. Int J Colorectal Dis. 2020;35(2):249-257.

7. Al-Mazrou AM, Chiuzan C, Kiran RP. Factors influencing discharge disposition after colectomy. Surg Endosc. 2018;32(7):3032-3040.

8. Mohanty S, Liu Y, Paruch JL, et al. Risk of discharge to postacute care: a patientcentered outcome for the american college of surgeons national surgical quality improvement program surgical risk calculator. JAMA Surg. 2015;150(5): 480-484.

9. Hyder JA, Wakeam E, Habermann EB, Hess EP, Cima RR, Nguyen LL. Derivation and validation of a simple calculator to predict home discharge after surgery. J Am Coll Surg. 2014;218(2):226-236.

10. Gadzinski AJ, Dimick JB, Ye Z, Zeller JL, Miller DC. Transfer rates and use of postacute care after surgery at critical access vs non-critical access hospitals. JAMA Surg. 2014;149(7):671-677.

11. Rahman M, McHugh J, Gozalo PL, Ackerly DC, Mor V. The contribution of skilled nursing facilities to hospitals' readmission rate. Health Serv Res. 2017;52(2): 656-675.

12. Balentine CJ, Mason MC, Richardson PJ, et al. Variation in postacute care utilization after complex surgery. J Surg Res. 2018;230:61-70.

13. Chow CJ, Gaertner WB, Jensen CC, Sklow B, Madoff RD, Kwaan MR. Does hospital transfer impact outcomes after colorectal surgery? Dis Colon Rectum. 2017;60(2):194-201.
14. Sharp SP, Ata A, Valerian BT, Canete JJ, Chismark AD, Lee EC. Complications and surgical outcomes after interhospital transfer vs direct admission in colorectal surgery: a National Surgical Quality Improvement Program analysis. Am J Surg. 2017;213(6):1031-1037.

15. Lucas DJ, Ejaz A, Haut ER, Spolverato G, Haider AH, Pawlik TM. Interhospital transfer and adverse outcomes after general surgery: implications for pay for performance. J Am Coll Surg. 2014;218(3):393-400.

16. McHugh JP, Foster A, Mor V, et al. Reducing hospital readmissions through preferred networks of skilled nursing facilities. Health Aff. 2017;36(9): 1591-1598.

17. Tyler DA, Gadbois EA, McHugh JP, Shield RR, Winblad U, Mor V. Patients are not given quality-of-care data about skilled nursing facilities when discharged from hospitals. Health Aff. 2017;36(8):1385-1391.

18. Jurt J, Hubner M, Pache B, Hahnloser D, Demartines N, Grass F. Respiratory complications after colorectal surgery: avoidable or fate? World J Surg. 2018:42(9):2708-2714.

19. Li L, Lau KS, Ramanathan V, et al. Ileostomy creation in colorectal cancer surgery: risk of acute kidney injury and chronic kidney disease. J Surg Res. 2017;210:204-212.

20. Zafar SN, Shah AA, Channa H, Raoof M, Wilson L, Wasif N. Comparison of rates and outcomes of readmission to index vs nonindex hospitals after major cancer surgery. JAMA Surg. 2018;153(8):719-727.

21. Werner RM, Coe NB, Qi M, Konetzka RT. Patient outcomes after hospital discharge to home with home health care vs to a skilled nursing facility. JAMA Intern Med. 2019;179(5):617-623.

22. Hutter MM, Rowell KS, Devaney LA, et al. Identification of surgical complications and deaths: an assessment of the traditional surgical morbidity and mortality conference compared with the American College of SurgeonsNational Surgical Quality Improvement Program. J Am Coll Surg. 2006;203(5): 618-624.

23. Key NS, Khorana AA, Kuderer NM, et al. Venous thromboembolism prophylaxis and treatment in patients with cancer: ASCO clinical practice guideline update. J Clin Oncol. 2020;38(5):496-520.

24. Kanters AE, Nikolian VC, Kamdar NS, Regenbogen SE, Hendren SK, Suwanabol PA. Academic hospitals discharge fewer patients to postacute care facilities after colorectal resection. Dis Colon Rectum. 2019;62(4):483-490. 